state that even though they would usually assign great disvalue to a false-positive result, their priority on this occasion is to avoid a false-negative result (or vice versa). Moreover, patients must be properly informed about the relevant concepts. Many patients are unfamiliar with the concept of over-diagnosis and therefore may be unable to weigh the relative risk of unnecessary diagnosis and treatment against the risk of failing to discover a cancer ${ }^{18}$. Moreover, patients may not always have preferences about such outcomes. There must still be a sensible default decision threshold that can be used in cases in which patients choose to withhold their attitudes or simply have no preferences.

There is also a danger of exaggerating the precision of the probabilities. If the dataset used to train the algorithm was small or non-representative, a probability range may be a more reasonable output than a precise probability. There is also a risk that clinicians will be unwilling or unprepared to take the patient's risk-value profile into account. These recommendations would create a more complex decision task for clinicians than reliance on a pre-programmed threshold; this emphasizes the importance of training in the use of $\mathrm{AI}$ in clinical settings and the co-design of diagnostic devices with physicians and patients.

These challenges notwithstanding, tailoring decision thresholds to the patient through the use of information about the patient's values and attitude about risk is vastly preferable to leaving them to be fixed by the software developer in a one-size-fits-all manner.

\section{Jonathan Birch (D) ${ }^{\boxplus}$, Kathleen A. Creel ${ }^{2}$, Abhinav K. Jha ${ }^{3}$ and Anya Plutynski (D) 4 ${ }^{I}$ Centre for Philosophy of Natural and Social Science, London School of Economics and Political Science, London, UK. ${ }^{2} \mathrm{McCoy}$ Family Center for Ethics in Society and the Institute for Human-Centered Artificial Intelligence, Stanford University, Stanford, CA, USA. ${ }^{3}$ Department of Biomedical Engineering and Mallinckrodt Institute of Radiology, Washington University in St. Louis, St. Louis, MO, USA. ${ }^{4}$ Department of Philosophy and Division of Biology and Biomedical Sciences, Washington University in St Louis, St Louis, MO, USA.

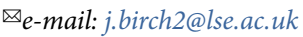

Published online: 31 January 2022

https://doi.org/10.1038/s41591-021-01624-y

References

1. McCradden, M. D. et al. Nat. Med. 26, 1325-1326 (2020).

2. Grote, T. \& Berens, P. J. Med. Ethics 46, 205-211 (2019).

3. Tschandl, P. et al. Nat. Med. 26, 1229-1234 (2020).
4. McKinney, S. M. et al. Nature 577, 89-94 (2020).

5iu, Z. et al. Phys. Med. Biol. 66, 124002 (2021).

6. Komorowski, M. et al. Nat. Med. 24, 1716-1720 (2018).

7. Turbé, V. et al. Nat. Med. 27, 1165-1170 (2021)

8. Claassen, J. et al. N. Engl. J. Med. 380, 2497-2505 (2019).

9. Hastie, T. The Elements of Statistical Learning: Data Mining, Inference, and Prediction (Springer, 2009).

10. Plutynski, A. in Exploring Inductive Risk: Case Studies of Values in Science (eds. Elliott, K. C. \& Richards, T.) 149-170 (Oxford University Press, 2017).

11. Douglas, H.E. Science, Policy, and the Value-free Ideal (University of Pittsburgh Press, 2009).

12. Bright, L. K. Synthese 195, 2227-2245 (2017).

13. Wenner, D. M. Int. J. Fem. Approaches Bioeth. 13, 28-48 (2020).

4. Buchak, L. J. Med. Ethics 43, 90-95 (2016).

15. Pan, C. H. \& Statman, M. J. Invest. Consult. 13, 54-63 (2012).

16. Ongena, Y. P. et al. J. Am. Coll. Radiol. 18, 79-86 (2021).

17. Birch, J., Creel, K., Jha, A. \& Plutynski, A. Zenodo https://doi.org/ 10.5281/zenodo.5589207 (2021).

18. Nagler, R. H. et al. Med. Care 55, 879-885 (2017)

\section{Acknowledgements}

We thank S. Bhalla, L. Kofi Bright, A. Houston, L. Hudetz, R. Short, J. Swamidass, K. Vredenburgh, Z. Ward, K. Wright and patient groups at Washington University in St Louis, Stanford University and Johns Hopkins University for their input and advice. A.K.J. acknowledges support from the National Institute of Biomedical Imaging and Bioengineering of the US National Institutes of Health (R01-EB031051 and R56-EB028287).

\section{Author contributions}

All authors contributed to conceptualization, methodology (survey design), investigation (consulting patients), and writing (review and editing). J.B. wrote the original draft.

The authors declare no competing interests.

\title{
The French health pass holds lessons for mandatory COVID-19 vaccination
}

The passe sanitaire increased levels of vaccination, but to a lower extent among the most vulnerable, and did not reduce vaccine hesitancy itself, showing the importance of outreach to underserved communities and the potential limits of mandatory vaccination policies.

\section{Jeremy K. Ward, Fatima Gauna, Amandine Gagneux-Brunon, Elisabeth Botelho-Nevers, Jean-Luc Cracowski, Charles Khouri, Odile Launay, Pierre Verger and Patrick Peretti-Watel}

$\mathrm{P}$ ublic authorities in many countries are considering mandating vaccination against COVID-19 for the whole eligible population ${ }^{1}$. Most countries are confronted with the difficulties of reaching the vaccination rates obtained for diseases such as measles, which are often above 95\%. During the summer of 2021, French authorities implemented a health pass, or passe sanitaire, requiring everyone aged 12 and older to present proof of vaccination or a negative test for SARS-CoV-2 to access a wide array of public spaces, including bars, libraries and hospitals. The introduction of the passe sanitaire markedly increased the number of people vaccinated against COVID-19. But, as of November 2021, coverage is plateauing at around $90 \%$ of the eligible population and a debate has arisen on whether the next step should be mandating this vaccination ${ }^{2}$.

There are lessons to be learnt from the French experience with the health pass that contribute to the current debate on mandatory COVID-19 vaccination.

\section{Barriers to vaccination}

In France, vaccination coverage against COVID-19 rose steadily during the first half of 2021 until it reached a first plateau in mid-June, with around $60 \%$ of the adult population having had at least one dose 2 . After having vaccinated the most willing, public authorities were confronted with three classic barriers to vaccination ${ }^{3}$ (Table 1 ). The first was doubts regarding the safety of vaccines against COVID-19,5. The proportion of the population who intended to receive the 


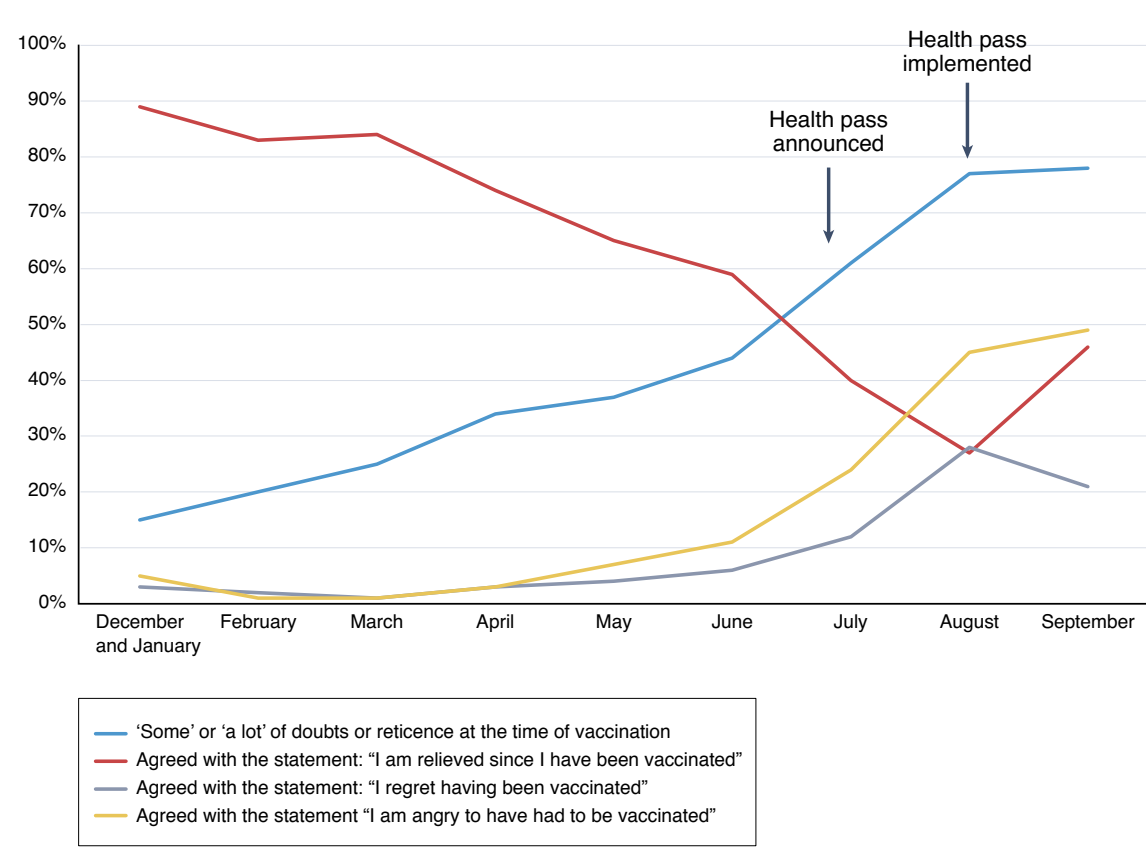

Fig. 1 | Doubts about vaccination among the vaccinated. French adults' experience of COVID-19 vaccination, depending on the month in which they received their first dose. The health pass was announced on 12 July 2021 and implemented on 9 August. $n=1,619$ respondents who had at least one dose of the COVID-19 vaccine out of a representative sample of 2,015 people aged $>18$ years; data collection: 22 September 2021 to 1 October 2021

vaccine had improved since December 2020, when $55 \%$ of the French adult population was unwilling to get the vaccine. Nevertheless, around one-quarter of the population was still unwilling in June $2021^{6,7}$.

The second barrier to vaccination was complacency, as many people did not perceive COVID-19 to be a serious threat to their personal health ${ }^{6}$. The third barrier was that even though COVID19 vaccines in France are free of charge, reaching those with less access to the healthcare system remained a problem. The main pathway to vaccination against COVID-19 was to book appointments via an online website, which therefore relied on the person's capacity to navigate the health system. Without a strong investment in local outreach operations, access for marginalized groups, including isolated older people, was limited. By mid-June 2021, only $70 \%$ of people aged 80 years or more had been fully vaccinated $^{2}$

\section{Increased uptake}

The health pass was announced on 12 July 2021 and implemented on 9 August, as a response to the Delta variant. To enter several public places, including restaurants, people aged 12 and over would have to present proof of full vaccination or a negative COVID-19 test performed in the previous $72 \mathrm{~h}$; vaccination was also made mandatory for healthcare workers. After this announcement, the share of the eligible population vaccinated with two doses increased from $49 \%$ on 12 July to $89 \%$ by mid-December 2021. An announcement that the health pass would require a booster dose by mid-January was also followed by a wave of appointment bookings.

Any vaccination policy that relies on constraints risks stoking anti-vaccine sentiments and vaccine hesitancy ${ }^{8,9}$. The health pass elicited strong criticism from opposition parties, as well as protests across France in early August attended by up to 240,000 people $^{10}$. Attendance at protests against the health pass has waned markedly, but the protests were still supported by around $30 \%$ of the population as of September $2021^{7,11}$.

Despite protests and opposition, public attitudes toward COVID-19 vaccination do not seem to have deteriorated. Intentions to vaccinate in mainland France continued to rise between June and July 2021, and have remained relatively stable since ${ }^{7}$.

However, vaccination rates have plateaued just above $90 \%$ among the eligible population in mainland France and remain low in several overseas departments and regions, such as Guadeloupe, Martinique and French Guiana, where less than $40 \%$ of the population has had at least one dose and opposition to the health pass is much stronger ${ }^{2,12}$.

\section{Vaccinating the hesitant and the marginalized}

The health pass is limited in its effectiveness, as although it has been effective in getting the complacent to book their vaccination appointments, it does not address all barriers to vaccination. For many people, the health pass had little effect, because the elderly, the poorest and the most marginalized do not engage much with the activities covered by the pass, such as eating out in restaurants. For instance, as of 12 October 2021, only $86 \%$ of over- 80 year olds had been fully vaccinated $^{2}$.

The health pass has encouraged vaccination of many who were hesitant or reluctant, but it has not reduced hesitancy itself. A survey from September 2021 found that $42 \%$ of vaccinated people were still reluctant or had doubts about the vaccine at the time of their first dose ${ }^{7}$. More importantly, the share of vaccinated people with doubts about the vaccine increased from $44 \%$ to $61 \%$ after the health pass was implemented (Fig. 1).

France remains a very vaccine-hesitant country, and this hesitancy does not seem to have decreased much as a result of either the COVID-19 health pass or mandatory infant vaccines, which were extended in 2017. One of the rationales for extending mandatory infant vaccination was that such a strong gesture would signal to the public the complete faith of authorities in these vaccines ${ }^{13}$. Although this policy did not elicit a public backlash, trust in vaccines does not seem to have significantly improved and France is still a very vaccine-hesitant country, as demonstrated in earlier stages of the pandemic ${ }^{4,5,14}$.

There is little to suggest that the health pass has convinced many skeptics about the benefits of this vaccination ${ }^{15}$ and there remains a small but considerable proportion (around 5-10\% of the population) who have decided not to take the vaccine for COVID-197. Vaccinating people who are hesitant or reluctant has potentially negative consequences, which can reinforce mistrust of institutions and of the healthcare system ${ }^{3,8,16}$.

A feeling of coercion while being vaccinated can cause a nocebo effect, in which negative outcomes occur because of a belief that the vaccine will harm them. The nocebo effect might explain why, in our survey, the share of vaccinated people who said they suffered side effects from the vaccine increased from $34 \%$ among those who had their first dose in June to $57 \%$ of those who had their first dose in August, after the health pass was implemented. 
Table 1 | Effects of the health pass in France on three dimensions of vaccine hesitancy

Reason for vaccine hesitancy
Complacency (low risk
perceptions, vaccination not
perceived as an urgent issue)

Confidence (trust in the safety and efficacy of the vaccines, in the health system and in policy-makers)

\section{Effects of health pass} Very effective in persuading the complacent to get vaccinated as the vaccine made it very convenient to maintain their normal activities.

Some reluctant people agreed to be vaccinated, despite their enduring doubts and hostility. Limited effect in overseas departments and regions where distrust of the state is greater.

The health pass was accompanied There have been insufficient by increased availability, including outreach efforts in underserved more appointments in vaccination communities to address barriers centers. to vaccination.
Limits of health pass

Limited effect on underserved people who do not use activities covered by the health pass, including older people, people on low incomes, migrants, and homeless people.

Many people remain unconvinced, with strong criticism from opposition parties, protests in metropolitan France, and social uprisings in several overseas departments and regions.

outreach efforts in underserved
Convenience (availability and accessibility of the vaccines, levels of health literacy, the appeal of immunization services)

\section{The importance of trust}

In France, opposition to vaccines against COVID-19 is grounded in a distrust of the mainstream political system and the current government in particular ${ }^{7,11}$. Some of the government's choices have not helped to build this trust. Trust is built by clearly explaining the uncertainties and constraints bearing on the decisions being made, so that the public can understand them, even when they change, and trust that they are based on the best available science ${ }^{17}$. Projecting certainty and control leads to avoidable changes in messages and the breaking of unnecessary promises.

For example, after the third lockdown, the French government required a negative COVID-19 test or proof of vaccination for large group activities, with a promise that the health pass would never include restaurants or cinemas ${ }^{18}$. This promise was broken just two months later. In November 2020, the President of France also promised that COVID-19 vaccination would never become mandatory ${ }^{19}$, a promise that has so far been kept, but that could damage trust if it were to be broken. Each time the government changed the rules, opposition parties seized the opportunity to score political points, leading to heated public debates. With the French presidential election in just a few months, mandatory vaccination policies could further politicize the COVID-19 vaccine.Making vaccination routine for everyone

Booster doses for COVID-19 are now being rolled out across high-income countries and may be required for years to come, especially for those most at-risk of severe disease. This continued threat will require vaccination for COVID-19 to become routine for many.

It is often thought that mandates are effective because of the threat of sanctions, but evidence suggests that their efficacy also rests elsewhere. With vaccine mandates, the responsibility for monitoring vaccination status and reminding people of the necessity to take the vaccine lies with key people who can easily reach the public ${ }^{20,21}$. For mandatory childhood vaccines, these tasks are usually performed by schools.

For mandatory vaccines for adults, there are no key people who are present in every adult's day-to-day life ${ }^{22}$. Enforcement could be done by employers, or alternatively by restaurants, bars and shops, as with the health pass. However, in France, such businesses were reluctant to enforce health norms and none of these actors are capable of reaching the people most at risk from COVID-19, including the unemployed and underserved communities.

Lessons from the COVID-19 health pass in France suggest that coercive measures such as mandatory vaccination can be effective in raising vaccination rates, but do not solve all of the issues that affect uptake. Mandatory vaccination for COVID-19 runs the risk of politicizing vaccination further and reinforcing distrust of vaccines, but national contexts will determine whether such a mandate is ethically justifiable or necessary to make COVID-19 vaccination routine. Regardless of whether vaccines are made mandatory or not, reaching high vaccine coverage requires a diversity of tools. Outreach programs and sustained efforts to motivate those who are hesitant should be the cornerstone of any COVID-19 vaccination policy.

\section{Jeremy K. Ward (1D) 1, ${ }^{\square}$, Fatima Gauna3 Amandine Gagneux-Brunon $n^{4,5,6,7}$, Elisabeth Botelho-Nevers ${ }^{4,5,6,7}$, \\ Jean-Luc Cracowski ${ }^{8}$, Charles Khouri ${ }^{8,9}$ Odile Launay ${ }^{7,10}$, Pierre Verger ${ }^{3,7}$ and Patrick Peretti-Watel ${ }^{2,3}$}

${ }^{1}$ CERMES3 (INSERM, CNRS, EHESS, Université de Paris), Villejuif, France. ${ }^{2}$ VITROME (Aix Marseille Université, IRD, AP-HM, SSA), Marseille, France. ${ }^{3}$ Observatoire régional de la santé PACA (ORS Paca), Aix-Marseille Université, Marseille, France. ${ }^{4}$ Centre International de Recherche en Infectiologie, Team GIMAP, Université de Lyon, Université Jean Monnet, Université Claude Bernard Lyon 1, Inserm, U111, Centre National de Recherche Scientifique (CNRS), Unité mixte de recherche (UMR) 530, Lyon, France. ${ }^{5}$ Inserm, CIC 1408 Vaccinologie, CHU de Saint-Etienne, Saint-Etienne, France. ${ }^{6}$ Chaire PREVACCI, Université Jean Monnet, Saint-Etienne, France. ${ }^{7}$ Inserm, F-CRIN, I-REIVAC/ COVIREIVAC, Paris, France. ${ }^{8}$ Pharmacovigilance Unit, Grenoble Alpes University Hospital, Grenoble, France. ${ }^{9}$ Univ. Grenoble Alpes, HP2 Laboratory, Inserm U1300,, Grenoble, France. ${ }^{10}$ Université de Paris, Inserm, Centre d'Investigation Clinique (CIC) 1417, Assistance Publique - Hôpitaux de Paris, CIC Cochin-Pasteur, Hôpital Cochin, Paris, France.

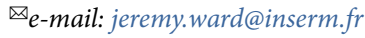

Published online: 12 January 2022 https://doi.org/10.1038/s41591-021-01661-7

References

1. Hannah, A. \& Attwell, K. https://theconversation.com/bidenannounces-covid-vaccine-mandate-for-100-million-americansaustralia-shouldnt-follow-just-yet-168066 (2021).

2. Santé Publique France. https://www.santepubliquefrance.fr/ dossiers/coronavirus-covid-19/coronavirus-chiffres-cles-etevolution-de-la-covid-19-en-france-et-dans-le-monde (accessed 11 December 2021).

3. Goldenberg, M. J. Vaccine Hesistancy (Univ. Pittsburgh Press, 2021

4. Ward, J. K. et al. Soc. Sci. Med. 265, 113414 (2020).

5. Schwarzinger, M. et al. Lancet Public Health 6, e210-e221 (2021).

6. Ward, J. K. et al. http://www.orspaca.org/sites/default/files/ enquete-COVIREIVAC-rapport.pdf (2021).

7. Ward, J. K. et al. http://www.orspaca.org/sites/default/files/NoteSLAVACO-Vague2.pdf (2021).

8. Omer, S. B. et al. Nature 571, 469 (2019).

9. Sprengholz, P. et al. Appl. Psychol. Health Well Being 13 986-995 (2021)

10. Bronner, L. https://www.lemonde.fr/planete/article/2021/09/11/ a-paris-le-noyau-dur-et-heterogene-de-la-contestation-contrele-passe-sanitaire_6094342_3244.html (2021).

11. Lévy, J.-D. et al. https://harris-interactive.fr/opinion_polls/ barometre-dintentions-de-vote-pour-lelection-presidentiellede-2022-vague-11/ (2021).

12. Le Monde-AFP. https://www.lemonde.fr/societe/article/2021/ 10/27/obligation-vaccinale-le-chu-de-guadeloupe-condamnedes-operations-commandos-menees-par-des-personnelsgrevistes_6099993_3224.html (2021).

13. Ward, J. K. et al. Vaccine 36, 14 (2018).

14. Lindholt, M. F. et al. BMJ Open 11, e048172 (2021).

15. Figueiredo de, A. et al. EClinicalMedicine 40, 101109 (2021).

16. Reich, J. A. Calling the Shots: Why Parents Reject Vaccines (NYU Press, 2016)

17. Petersen, M. B. Nature 598, 237 (2021). 
18. Le Parisien-AFP. https://www.leparisien.fr/politique/leveeprogressive-des-restrictions-sanitaires-lexamen-du-projetde-loi-par-les-deputes-sannonce-anime-10-05-2021-SF4Y5TK 6HBBCFPPLAL5IJXRVGU.php (2021).

19. Macron, E. https://www.elysee.fr/emmanuel-macron/2020/11/ 24/adresse-aux-francais-24-novembre (2020).

20. McCoy, C. J. Health Polit. Policy Law 44, 6 (2019).

21. Attwell, K. et al. Milbank Q. 97, 4 (2019).

22. Gostin, L. O. et al. J. Am. Med. Assoc. 325, 6 (2021).

Acknowledgements

This research benefited from the financial support of the Agence Nationale de la Recherche (ANR SLAVACO: 20-COV8-0009-01) and the Institut national de la santé et de la recherche médicale (INSERM), the Ministry of Health, and The Ministry of Higher Education and Research via the CORIVEIVAC platform (intended for COVID-19 vaccine clinical research). The funding sources had no role in the design of the study, analysis of the data or writing of the paper. We thank C. Bérenger, S. Cortaredona, L. Fressard and G. Maradan for data collection and analysis.

Author contributions

Conception or design of the work: J.K.W., P.P.W., F.G., A.G.B., E.B.N., J.-L.C., C.K., O.L. and P.V. Acquisition of the data: P.V. and J.K.W. Analysis: J.K.W., F.G., P.P.W.
Interpretation of data for the work: J.K.W., P.P.W., F.G., A.G.B., E.B.N., J.-L.C., C.K., O.L. and P.V.

Competing interests

J.K.W. is a member of the Commission Technique des Vaccinations at the Haute Autorité de la Santé, O.L. is a member of the Comité scientifique sur les vaccins COVID-19, P.P.W. is a member of the Conseil d'Orientation de la Stratégie Vaccinale, and J.L.C. is responsible for the Drug and COVID working group of the French Pharmacological Society. The views expressed in this article are those of the authors, not these committees. 